\title{
La crue du Rhin de mai-juin 1999
}

\author{
The Rhine flood of May-June 1999
}

\author{
par Martial Gerlinger \\ Ingénieur des Ponts et Chaussées \\ chargé de l'Arrondissement Fonctionnel du Service de la Navigation de Strasbourg
}

The recent Rhine flood (May 12th-first days of June 1999) was essentially due to important quantities of winter snow. The stream flows and the duration were really exceptional. The dikes in the upper part of the Rhine (which is trained on almost all the french-german part) and the existant impounding water means permitted to limit the consequences. The present paper describes the flood management.

\section{I —HISTORIQUE DE L'AMÉNAGEMENT DU RHIN SUPÉRIEUR}

Les différentes phases d'aménagement sont présentées en annexe 1.

\subsection{La correction}

L'aménagement du Rhin Supérieur remonte essentiellement au XIX ${ }^{\mathrm{c}}$ siècle. Le Rhin était resté jusqu'en 1840 un fleuve à l'état sauvage, au lit parsemé d'îles souvent très étendues séparées par une succession de chenaux et de bras morts, et s'étendant au total sur une largeur de 2 à $3 \mathrm{~km}$. A chaque crue, de nouvelles îles se formaient, d'autres disparaissaient et le cours du fleuve était souvent modifié. Les différentes digues protégeant la plaine étaient fréquemment emportées et la navigation n'y était pas facile.

Cette situation amena les Etats riverains à envisager une protection efficace des villages contre les effets des crues et une meilleure définition du lit du Rhin. Le seul moyen semblait être d'enrayer la formation des îles et de rassembler le débit moyen du fleuve dans un lit unique. La correction du fleuve, entre Sonderheim et Bâle, planifiée par le Colonel badois Tulla, fut menée de 1840 à 1860 . Les travaux ont modifié la physionomie du fleuve en l'endiguant entre deux berges distantes de 200 à $250 \mathrm{~m}$ avec un tracé formé de tronçons rectilignes et de courbes d'un rayon minimal de $1000 \mathrm{~m}$. Les berges n'étaient plus submergées que pour des crues moyennes de débit $2000 \mathrm{~m}^{3} / \mathrm{s}$ (débit moyen du Rhin $=1000 \mathrm{~m}^{3} / \mathrm{s}$ ), le débordement étant limité sur les deux rives par un système continu de Digues des Hautes Eaux (DHE), situées parfois à plus d'un kilomètre en retrait.
Ces travaux ont effectivement amélioré nettement la protection contre les crues et la navigation au départ mais ont eu pour conséquence une forte augmentation de la vitesse d'écoulement de l'eau et des phénomènes d'érosion qui ont rendu la navigation difficile.

\subsection{La canalisation}

La canalisation entreprise au $\mathrm{xx}^{\mathrm{e}}$ siècle a permis de supprimer les inconvénients ci-dessus. La mise en service des usines hydroélectriques sur le Rhin Supérieur a démarré à partir de 1932 sur le Grand Canal d'Alsace. A l'issue des accords franco-allemands de 1956, un nouveau type d'aménagement est retenu, avec la réalisation de dérivations du Rhin sur lesquelles ont été construites les usines et les écluses (aménagement dit en feston). Quatre chutes ont ainsi successivement été mises en service entre Brisach et Strasbourg.

Compte tenu de la reprise de l'érosion à l'aval de la dernière chute, qui risquait d'avoir un effet néfaste sur les ports de Strasbourg et de Kehl et sur la nappe phréatique, et vu les intérêts multiples liés à la poursuite de la canalisation, deux chutes ont été construites dans le lit même du Rhin à l'aval de Strasbourg (Gambsheim et Iffezheim). La situation actuelle est présentée en annexe 2.

Les 8 chutes à l'amont de Strasbourg sont gérées par EDF; les 2 chutes suivantes à l'aval de Strasbourg sont gérées par les Etats (Services de la Navigation) à l'exception des usines hydroélectriques et des barrages mobiles qui sont concédés. 


\section{QMESURES DE RÉTENTION DES CRUES}

\subsection{Effet de l'aménagement du Rhin Supérieur}

Les aménagements du Rhin décrits ci-dessus et en particulier la canalisation, s'ils ont permis de protéger les riverains des secteurs canalisés contre des crues de fréquence supérieure à 1000 ans, ont également eu pour conséquence d'aggraver les crues à l'aval. Ainsi, une crue bicentennale à l'aval du secteur canalisé avait avant la canalisation un débit de l'ordre de $5000 \mathrm{~m}^{3} / \mathrm{s}$. Après la canalisation, si aucune mesure n'était engagée, le débit de la crue bicentennale serait de $5700 \mathrm{~m}^{3} / \mathrm{s}$.

Les Digues des Hautes Eaux (DHE) à l'aval de la dernière chute du Rhin d'Iffezheim ayant été dimensionnées pour la crue bicentennale avant la canalisation, il a été décidé d'engager des mesures pour retrouver l'objectif de protection contre les crues à l'aval contre une crue bicentennale.

\subsection{Mesures projetées}

\subsubsection{Convention franco-allemande du 6 décembre 1982}

Pour atteindre cet objectif et après examen des différentes solutions, la solution adoptée est celle qui consiste à retenir, au moment de la pointe de crue, le volume excédentaire entre Bâle et Worms à l'aide d'une combinaison de mesures se situant essentiellement entre Bâle et Iffezheim.

Les mesures suivantes ont été étudiées :

- Mancuvres exceptionnelles des usines EDF :

Par une manæuvre appropriée des usines EDF, il est en effet possible de créer un volume de rétention dynamique de 45 millions de $\mathrm{m}^{3}$.

- Utilisation des 2 barrages agricoles existants sur le VieuxRhin.

Il s'agit du barrage agricole de Brisach et du barrage agricole de Strasbourg/Kehl. L'écrêtement des crues est obtenu par un rehaussement de la cote de rétention aux barrages agricoles.

- Zones de rétention latérales dites "polders » :

zones endiguées latérales au Rhin pouvant être inondée au passage de la crue.

De nombreuses combinaisons des mesures évoquées cidessus ont été testées sur le modèle mathématique pour évaluer leur efficacité. La plus appropriée pour atteindre l'efficacité recherchée a été reprise dans la convention franco-allemande du 6 décembre 1982.

\subsubsection{Mesures projetées actuellement dans le cadre de la convention}

Les mesures de rétention sont présentées en annexe 3. Côté français ces mesures sont les suivantes:

- Mancuvres exceptionnelles des usines EDF.

- Polder de la Moder (5,6 $\mathrm{Mm}^{3}, 240$ hect.), opérationnel depuis 1992.

- Polder d'Erstein (7,8 $\mathrm{Mm}^{3}, 600$ hect.), dont la construction a démarré en 1997. Le plan de cette mesure est présenté en annexe 4 à titre d'exemple.

Le Land Bade-Wurtemberg réalise 13 mesures de rétention pour un volume de $168 \mathrm{Mm}^{3}$ et le Land RhénaniePalatinat 8 mesures pour un volume de rétention d'environ $50 \mathrm{Mm}^{3}$.

\subsubsection{Situation actuelle}

Les mesures de rétention actuellement opérationnelles sur le Rhin Supérieur sont les suivantes :

- Manœuvres exceptionnelles des usines EDF.

- Polders d'Altenheim.

- Barrage agricole de Strasbourg/Kehl (pas encore à la cote de rétention maximum pour l'instant, des mesures d'adaptation pour assurer le rabattement de la nappe dans les zones latérales étant actuellement en cours de réalisation).

- Polder de la Moder.

\section{III —LA GESTION DE LA CRUE}

\subsection{La crue du Rhin}

La crue du Rhin de mai-juin 1999 a été exceptionnelle tant par les débits atteints que par sa durée.

La première onde de crue (et également la plus importante) est arrivée à Bâle le 12 mai aux environs de 23 heures avec un débit de pointe de $4700 \mathrm{~m}^{3} / \mathrm{s}$ à Rheinfelden (soit environ $5000 \mathrm{~m}^{3} / \mathrm{s}$ à Bâle), correspondant à une crue de fréquence supérieure à 100 ans à ce niveau.

Les affluents alsaciens et du Bade Wurtemberg n'étant heureusement pas en situation de hautes eaux (ce qui est exceptionnel vu la situation météorologique dégradée sur l'ensemble de la Suisse), le débit de l'onde de crue ne s'est pas accentué sur le secteur franco-allemand pour arriver avec un débit de $4570 \mathrm{~m}^{3} / \mathrm{s}$ à Maxau (à l'aval de la frontière franco-allemande), soit une fréquence de retour de l'ordre de 50 ans.

Après une semaine avec des débits très soutenus de l'ordre de $3000 \mathrm{~m}^{3} / \mathrm{s}$, une seconde onde de crue est ensuite apparue à Bâle dans l'après-midi du 22 mai 1999 . L'ensemble des lacs suisses et en particulier le lac de Constance étant déjà remplis, un phénomène de "goutte d'eau " sur l'ensemble de la partie Est du bassin alpin du Rhin a conduit à une nouvelle onde de l'ordre de $4000 \mathrm{~m}^{3} / \mathrm{s}$ à Bâle (fréquence de retour d'environ 20 ans) supérieure à la valeur atteinte lors de la crue de février 1999.

Par la suite et jusqu'à mi-juin environ, les débits sont restés soutenus de l'ordre de $2500 \mathrm{~m}^{3} / \mathrm{s}$ à $3000 \mathrm{~m}^{3} / \mathrm{s}$ ( 3 fois le débit moyen) du fait du phénomène de vidange progressive des lacs suisses et de pluies fréquentes.

\section{- 3.2 Conséquence de la crue et organisation mise en place}

Les principales conséquences de cette crue exceptionnelle ont été des inondations importantes des îles du Rhin (entre le Vieux-Rhin et les canaux de dérivation), la mise en charge importante des Digues des Hautes Eaux (DHE) sur $18 \mathrm{~km}$ à l'aval du secteur canalisé et l'interruption de la navigation.

\subsubsection{Organisation mise en place}

Ces conséquences ont justifié la mise en alerte permanente pendant toute la crue du Centre d'Alerte Rhénan et d'Information Nautique de Gambsheim (CARING) du Service de la Navigation, et du centre d'alerte d'EDF à Kembs (Poste Hydraulique de Vallée, PHV) et la mise en place aux deux préfectures de département (Haut-Rhin et Bas-Rhin) d'une cellule de crise durant le passage de la première onde de crue.

Le Service de la Navigation de Strasbourg assure l'annonce de crue sur le Rhin franco-allemand, la gestion de la navigation, la gestion des polders français (pour le compte 
de Voies Navigables de France) et la gestion du Rhin à l'aval de Strasbourg en relation avec les concessionnaires hydroélectriques.

EDF assure en ce qui la concerne la gestion de l'ensemble des ouvrages sur le Rhin des 8 biefs à l'amont de Strasbourg et en particulier la mise en œuvre des manœuvres exceptionnelles des usines hydroélectriques.

Les échanges sur les prévisions des crues et la mise en œuvre des mesures de rétention des crues se font en relation avec plusieurs services :

- EDF (en particulier le Poste Hydraulique de Vallée à Kembs, PHV Kembs). EDF dispose d'un modèle de prévision des crues à court terme pour Kembs.

- Le service hydrologique et géologique national suisse pour les prévisions des crues à Bâle (cf. annexe 5).

- Les services de prévisions de crues et de gestion des mesures de rétention des crues du Land Bade Wurtemberg (cf. annexe 6) et du Land Rhénanie Palatinat.

- Le Service de la Navigation allemand de Fribourg.

Parallèlement à ces échanges, le CARING transmet les informations aux préfectures concernées (Bas-Rhin et HautRhin) ainsi qu'aux maires, services et particuliers concernés. Un exemple de message diffusé est transmis en annexe 7. Après autorisation par la préfecture, le CARING assure également une information régulière des médias.

\subsubsection{Inondations des îles du Rhin}

Les îles du Rhin sont très fréquentées par les promeneurs et les pêcheurs. Quelques habitations y sont également situées. Il importe donc de faire évacuer les zones inondables et le cas échéant les habitations qui pourraient se trouver isolées.

Cela est réalisé par la gendarmerie et les pompiers. Un survol en hélicoptère est également lancé par les services de la protection civile sur l'ensemble des îles du Rhin et le polder de la Moder préalablement aux inondations et en fonction des prévisions de crues.

\subsubsection{Surveillance des Digues des Hautes Eaux (DHE)}

Le secteur à l'aval d'Iffezheim (secteur non canalisé sur environ $18 \mathrm{~km}$ en rive française) est protégé par une Digue des Hautes Eaux (DHE) contre une crue de fréquence 200 ans environ. Pour vérifier la stabilité des digues qui sont mises en charge, la loi locale de 1891 permet de réquisitionner des équipes communales d'habitants des 5 villages concernés.

\subsubsection{Interruption de la navigation}

Durant la crue de mai-juin, la navigation a été arrêtée sur le secteur à courant libre à l'aval d'Iffezheim (secteur où la crue est la plus pénalisante pour la navigation) au total 27 jours, dont 19 jours d'affilé au départ. Cette interruption est exceptionnelle dans le mesure où jusqu'à présent, les interruptions les plus longues relevées étaient de l'ordre de 18 jours au total sur une année, avec au maximum 12 jours d'affilé.

Cette interruption a nécessité une gestion individuelle par le CARING de l'ensemble des bateaux (entre 100 et 200) présent sur le secteur et dans certains cas, un ravitaillement en eau de certains bateaux stoppés en dehors d'un port.

\subsection{La gestion de l'écrêtement de la crue}

Sur les mesures de rétention des crues disponibles actuellement (cf. paragraphe 2.3), l'ensemble des mesures de rétention a été mise en œuvre lors du passage de la première onde de crue le 13 mai 1999, à l'exception du polder de la Moder (comme cela avait déjà été le cas durant la crue de février 1999). Au total, un volume de rétention de l'ordre de 60 millions de $\mathrm{m}^{3}$ a ainsi pu être stocké.

Les critères de mise en œuvre des rétentions avaient été déterminés au préalable par un groupe de travail franco-allemand pour garantir une cohérence de la gestion de l'ensemble des mesures et pour éviter des mises en cuvres arbitraires. Les critères, qui sont des critères fixes comme par exemple le fait d'atteindre un certain débit à une échelle de référence, doivent permettre de répondre à l'ensemble des situations qui peuvent se présenter. Une série d'une vingtaine de crues historiques permettent ainsi des simulations pour optimiser les critères pour la crue bicentennale qui est notre objectif de protection.

Durant cette crue, le polder de la Moder n'a pas été mis en œuvre alors que le critère de mise en œuvre était pourtant atteint. Ce polder se trouvant tout à fait en aval des mesures de rétention existantes, nous avons en fait décidé de le garder en réserve après le passage de la première onde de crue dans la mesure où à ce moment là une seconde onde de crue de débit de pointe similaire était alors annoncée et où toutes les autres mesures de rétention avaient déjà été mises en œuvre. Au final, la seconde onde a été moins importante que prévu.

Au total, les mesures de rétention ont permis d'écrêter la crue de l'ordre de $400 \mathrm{~m}^{3} / \mathrm{s}$. Ainsi, sans mise en œuvre des mesures de rétention, le débit de $5000 \mathrm{~m}^{3} / \mathrm{s}$ (situation très critique vis-à-vis des Digues de Hautes Eaux) aurait été atteint à l'aval d'Iffezheim. 


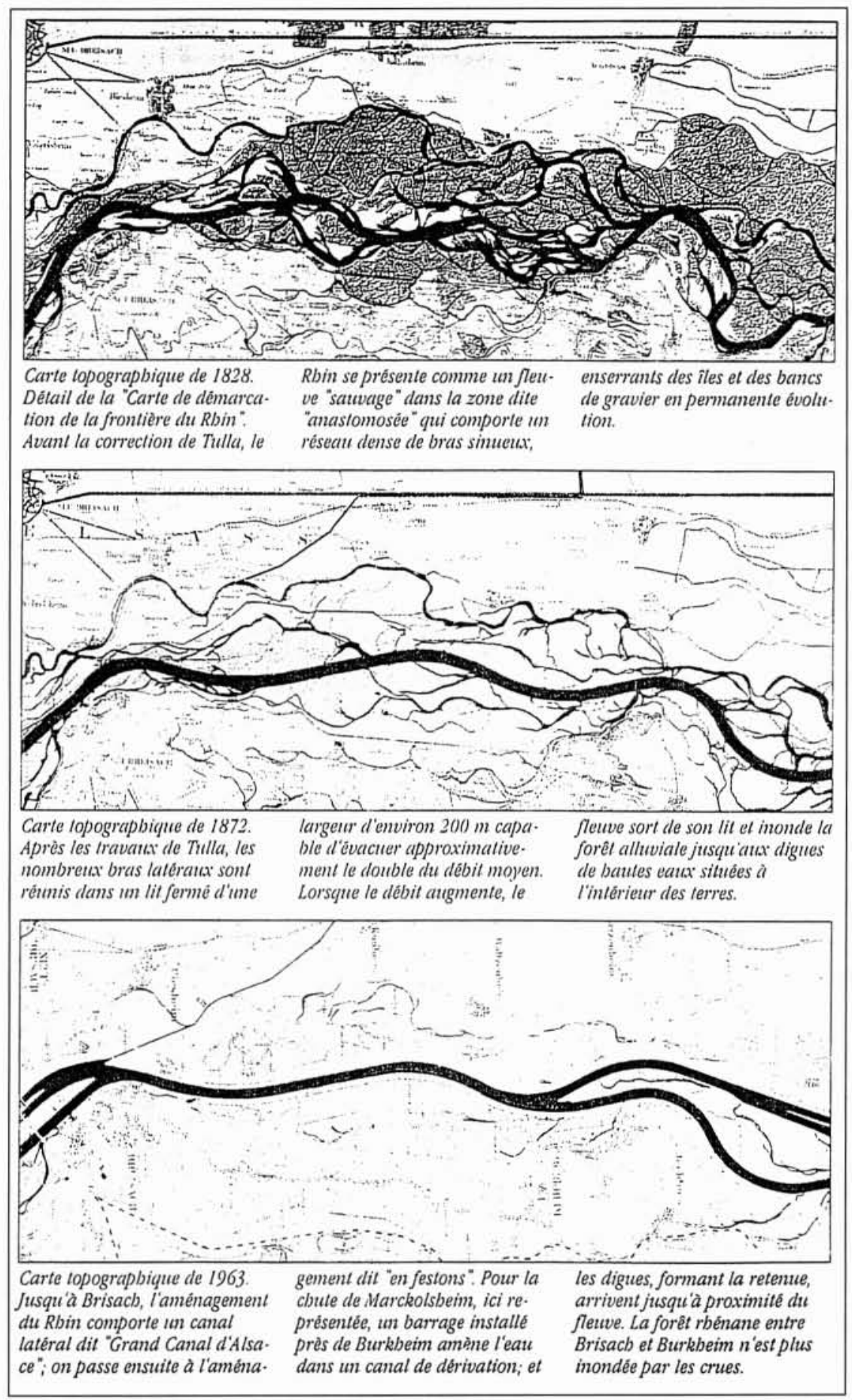

Annexe 1 : Aménagement du Rhin Supérieur 


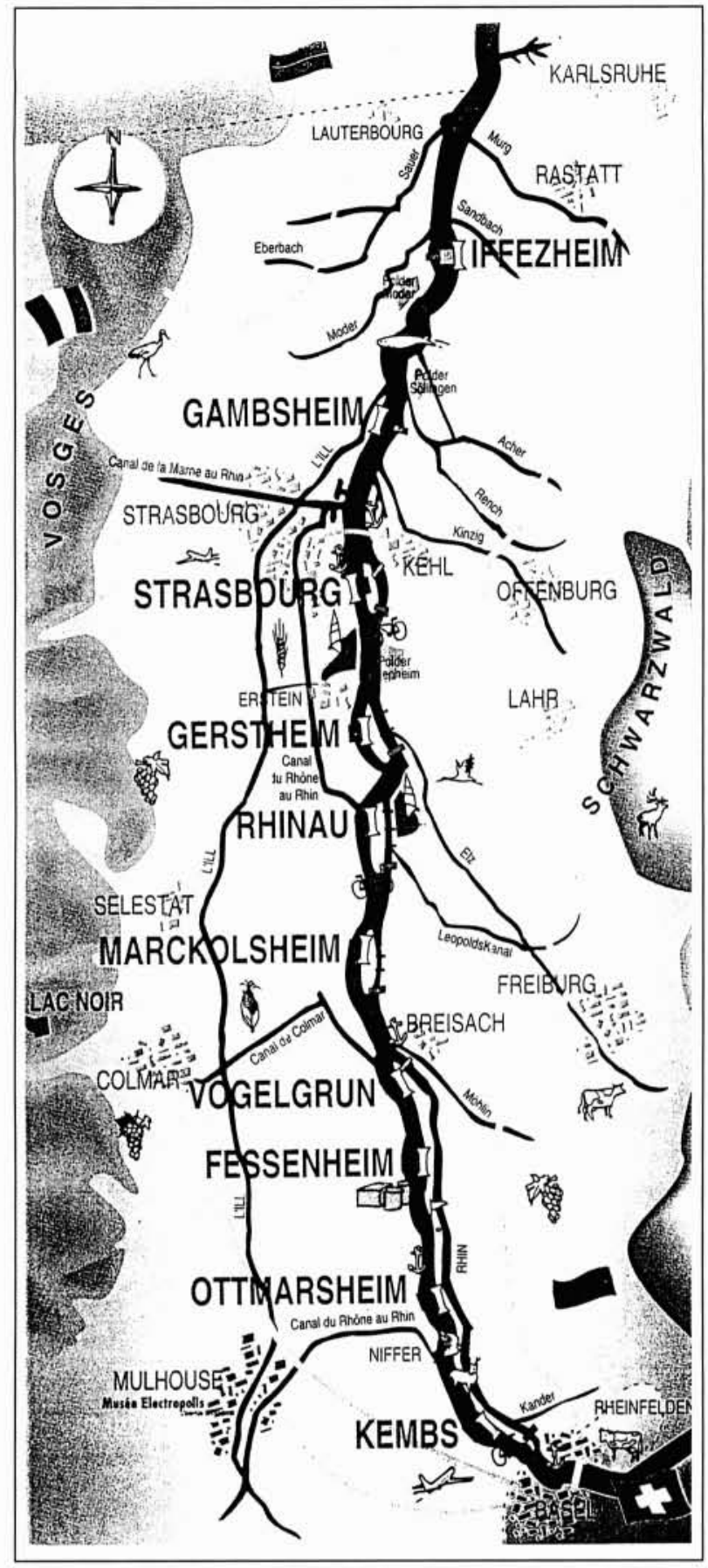

Annexe 2 : Situation actuelle 


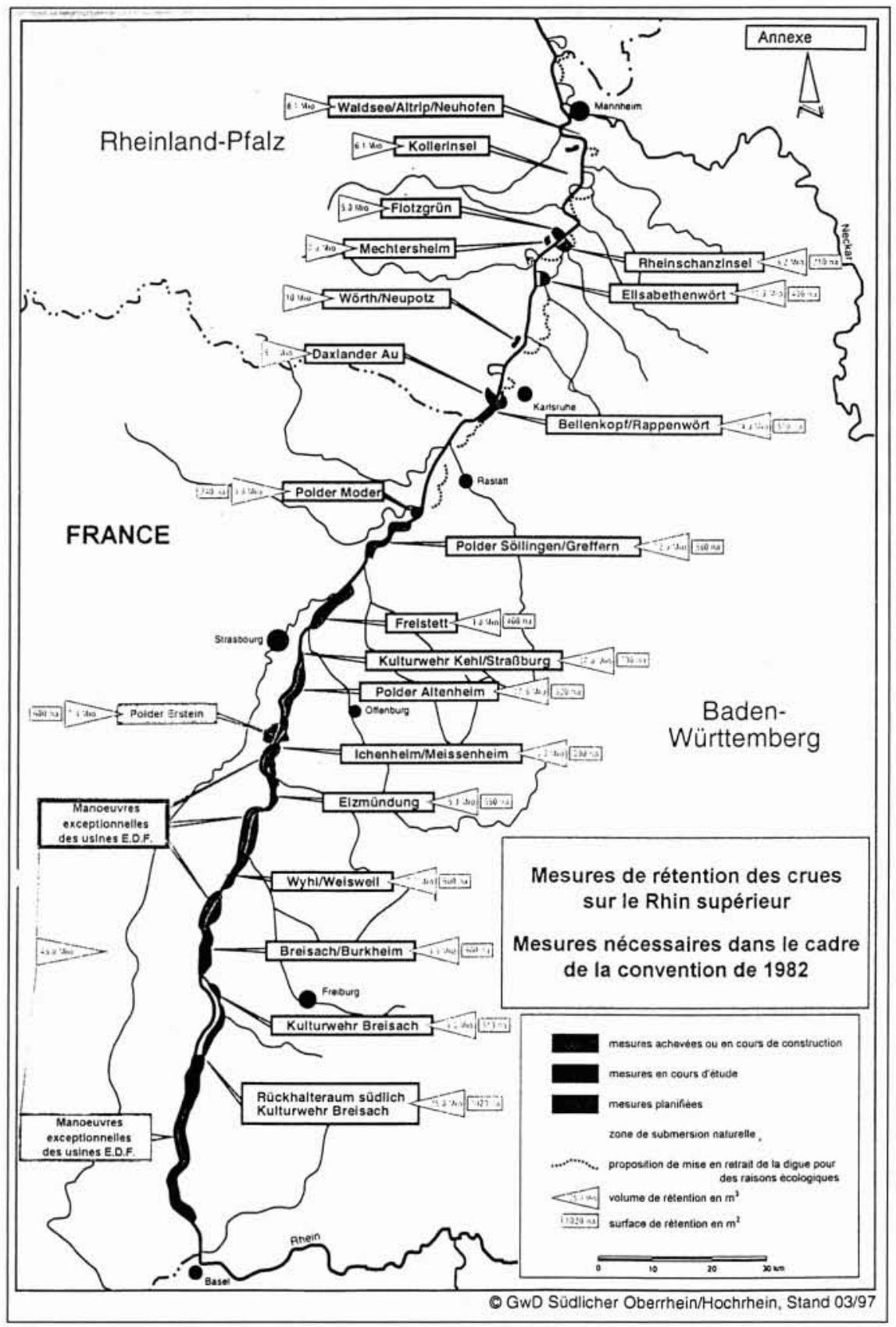

Annexe 3 : Les mesures de rétention des crues prévues sur le Rhin supérieur 


\begin{tabular}{|c|c|c|c|}
\hline \multicolumn{4}{|c|}{ Espaces de rétention sur le Rhin supérieur } \\
\hline Espace de rétention & $\begin{array}{l}\text { Type de } \\
\text { rétention }{ }^{2)}\end{array}$ & \begin{tabular}{|} 
D'après plan \\
de 1982 \\
[millions \\
de $\mathrm{m}^{3}$ ]
\end{tabular} & $\begin{array}{l}\text { D'après plan } \\
\text { actuel } \\
\text { [millions } \\
\text { de } \mathrm{m}^{3} \text { ] }\end{array}$ \\
\hline $\begin{array}{l}\text { France: } \\
\text { - manoeuvres except. des usines du Rhin "1 } \\
\text { Erstein } \\
\text { - Moder }\end{array}$ & $\begin{array}{l}\text { manoeuvres } \\
\text { polder } \\
\text { polder }\end{array}$ & $\begin{array}{r}45 \\
6 \\
5\end{array}$ & $\begin{array}{l}45 \\
7,8 \\
5,6\end{array}$ \\
\hline Total France & & 56 & 58,4 \\
\hline $\begin{array}{l}\text { Bade-Wurtemberg: } \\
\text { au sud de I'usine de Vieux-Brisach } \\
\text { barrage agricole de Vieux-Brisach " } \\
\text { Vieux-Brisach/Burheim } \\
\text { WhylMeisweil } \\
\text { débouché de l'Elz } \\
\text { Ichenheim/Meißenheim } \\
\text { - Altenheim } \\
\text { - barrage agricole de KehVStrasbourg " } \\
\text { Freistett } \\
\text { Söllingen/Greffern } \\
\text { Bellenkopt/Rappenwört } \\
\text { Elisabethenwört } \\
\text { lle de Rheinschanz }\end{array}$ & \begin{tabular}{|l} 
barrage ou \\
décaissement \\
barrage \\
polder \\
polder \\
polder \\
polder \\
polder \\
barrage \\
polder \\
polder \\
polder ou recul \\
de digues \\
polder ou recul \\
de digues \\
polder
\end{tabular} & $\begin{array}{c}53 \\
10 \\
\vdots \\
: \\
18 \\
37 \\
8 \\
8 \\
-\end{array}$ & $\begin{array}{r}25,0 \\
9,3 \\
9,5 \\
6,5 \\
7,7 \\
5,3 \\
5,8 \\
17,6 \\
37,0 \\
9,0 \\
12,0 \\
14,0 \\
11,9 \\
\\
6,2\end{array}$ \\
\hline Total Bade-Wurtemberg & & 126 & 167,3 \\
\hline $\begin{array}{l}\text { Rhénanie-Palatinat: } \\
\text { localisation non déterminée } \\
\text { - Daxlander Au } \\
\text { Wörth/Jockgrim } \\
\text { Neupotz } \\
\text { Mechtersheim } \\
\text { Flotzgrün } \\
\text { lle de Koller } \\
\text { Waldsee/Altrip/Neuhofen } \\
\text { Petersau/Bannen } \\
\text { Mittelbusch (Worms II) } \\
\text { Bodenheim/Laubenheim } \\
\text { Ingelheim }\end{array}$ & $\begin{array}{l}\text { polder } \\
\text { recul de digues } \\
\text { polder } \\
\text { polder } \\
\text { polder } \\
\text { polder } \\
\text { polder } \\
\text { recul de digues } \\
\text { recul de digues } \\
\text { poider } \\
\text { polder }\end{array}$ & $30+14$ & $\begin{array}{l}5,1 \\
8,8 \\
8,6 \\
7,4 \\
5,0 \\
6,1 \\
8,1 \\
1,4 \\
2,3 \\
6,0 \\
3,8\end{array}$ \\
\hline Total Rhénanie-Palatinat & & 44 & 62,6 \\
\hline Somme de tous les totaux: & & 226 & env. 288 \\
\hline $\begin{array}{ll}\text { - } & \text { Espaces de rétention achevés et opéra } \\
& \text { KehVStrasbourg encore limitée à env. } \\
\text { 1): } & \text { Les valeurs indiquées sont des maxim } \\
\text { 2): } & \text { Lefficacité de toutes les mesures dan } \\
& \text { dépend du débit du Rhin }\end{array}$ & $\begin{array}{l}12 \text { millions de } m^{3} \\
\text { ums; le volume rée } \\
\text { la partie non rég. }\end{array}$ & $\begin{array}{l}\text { He rétention au bar } \\
\text { à l'heure actuelle } \\
\text { l utilisé dépend du } \\
\text { ularisée du Rhin en }\end{array}$ & $\begin{array}{l}\text { rage agricole de } \\
\text { débit du Rhin } \\
\text { aval d'Iffezheim }\end{array}$ \\
\hline
\end{tabular}

Annexe 3 : Les mesures de rétention des crues prévues sur le Rhin supérieur 


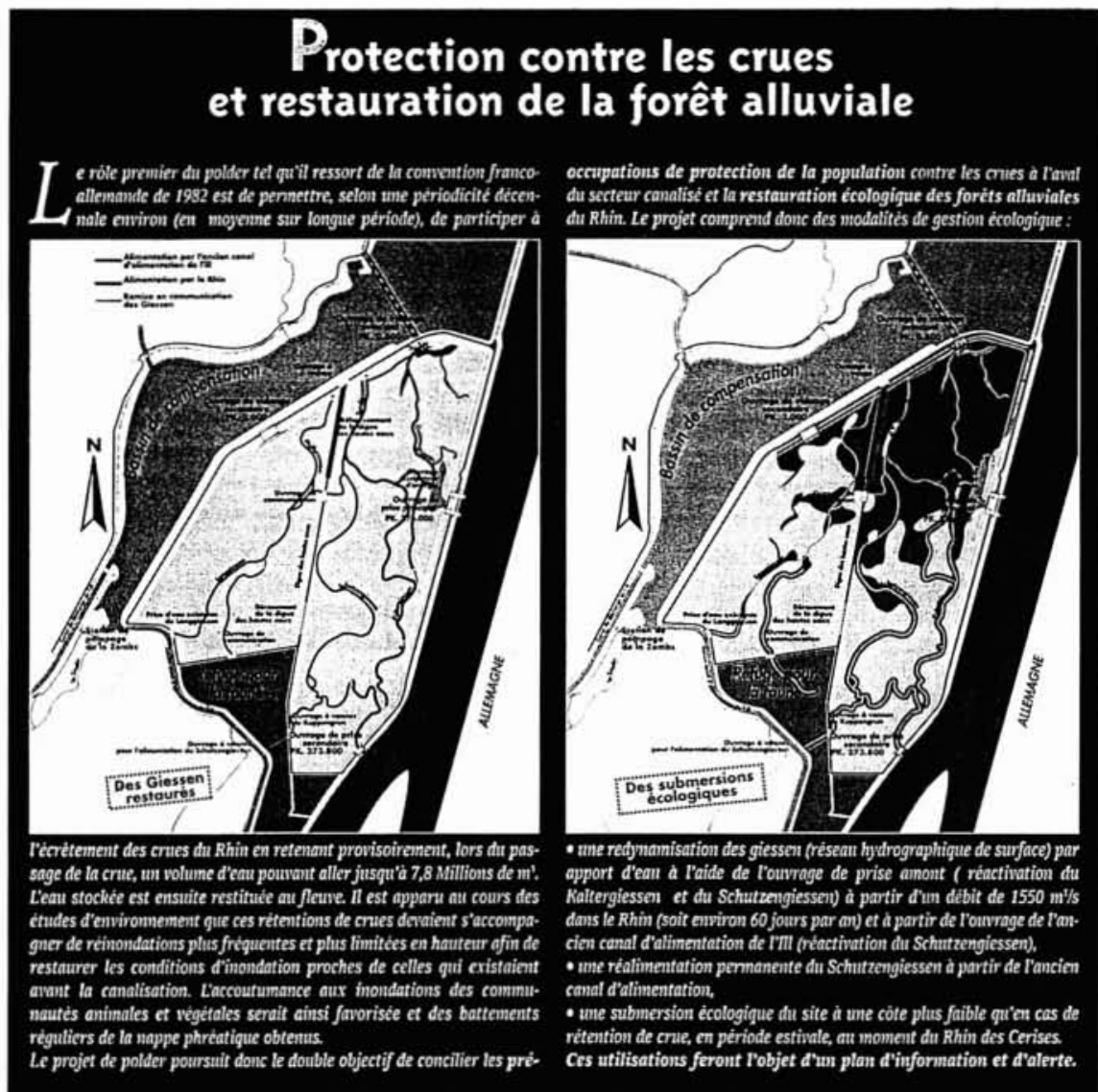

\section{Schéma de fonctionnement global}

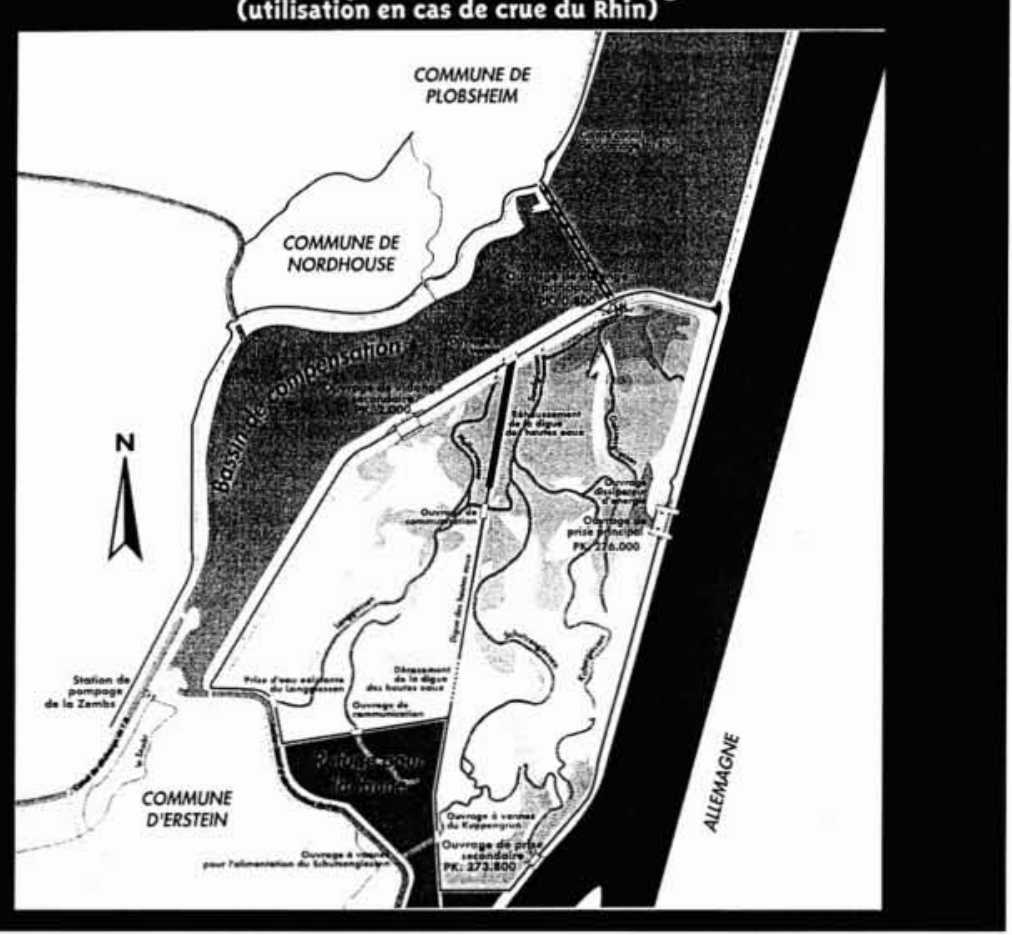

Anexe 4: Le Polder d'Erstein 


\section{Eau : ressources, aménagements, environnement}

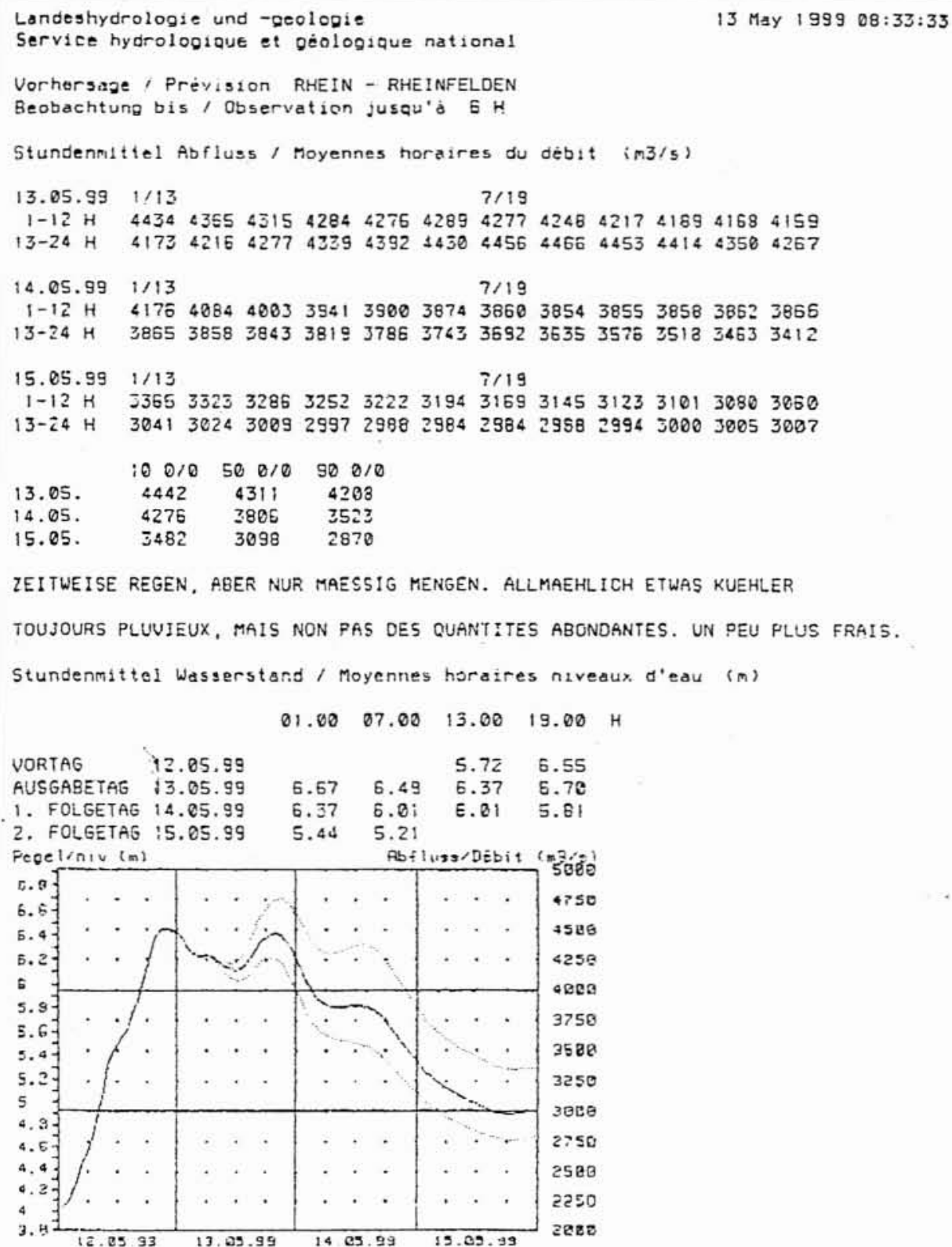

Annexe 5 : Prévision des crues suisses à Rheinfelden (amont de Bâle) 


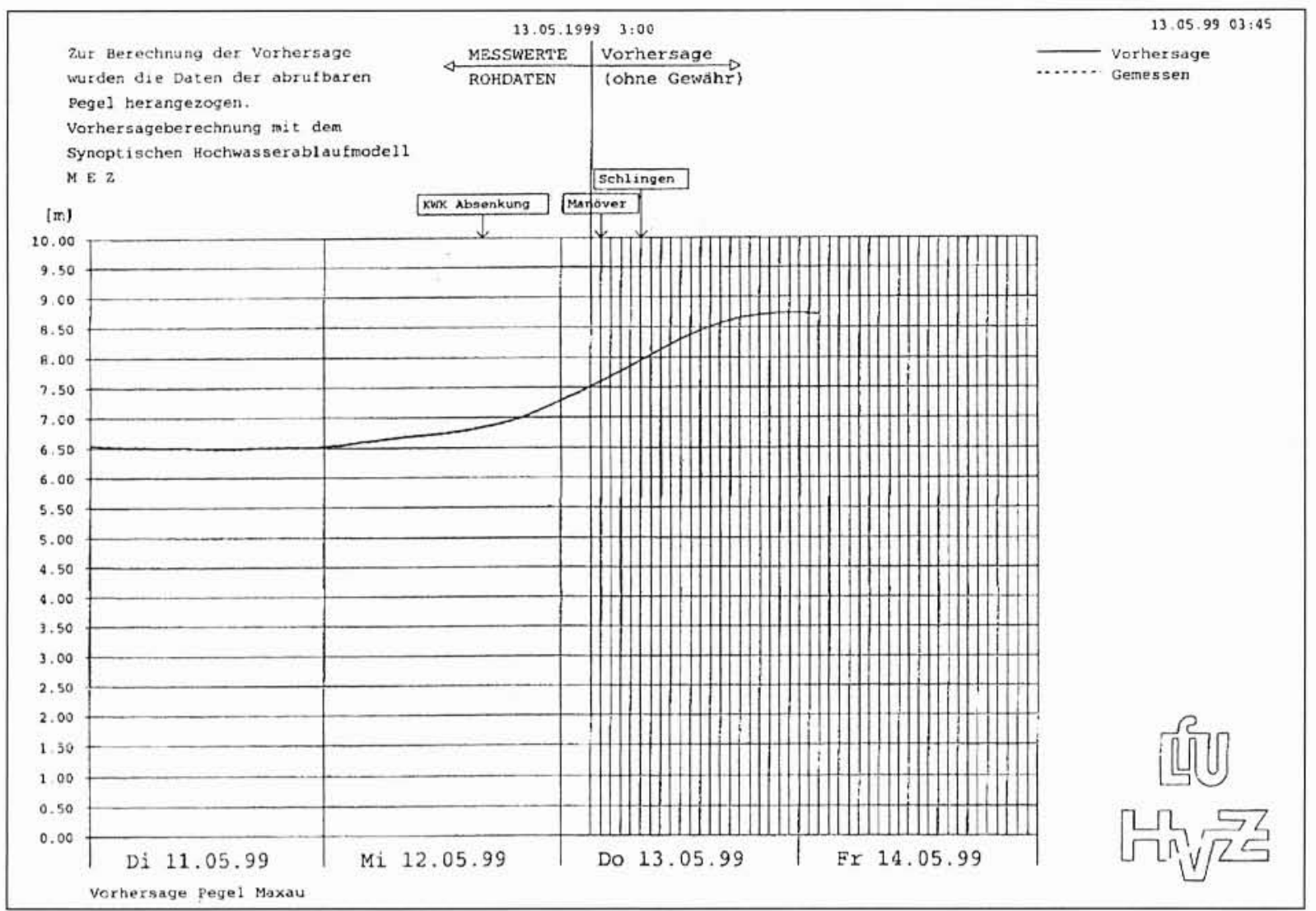

Annexe 6 : Prévision à 12 h par la Land Bade Wurtemberg à MAXAU (aval d'Iffezheim) 